\title{
Importance of dispersion in density functional calculations of cesium chloride and its related halides
}

\author{
F. Zhang, ${ }^{1, *}$ J. D. Gale, ${ }^{1}$ B. P. Uberuaga, ${ }^{2}$ C. R. Stanek, ${ }^{2}$ and N. A. Marks ${ }^{3}$ \\ ${ }^{1}$ Nanochemistry Research Institute, Department of Chemistry, Curtin University, P.O. Box U1987, Perth, Western Australia 6845, Australia \\ ${ }^{2}$ Material Science and Technology Division, Los Alamos National Laboratory, Los Alamos, New Mexico 87545, USA \\ ${ }^{3}$ Nanochemistry Research Institute, Discipline of Physics and Astronomy, Curtin University, P.O. Box U1987, Perth, \\ Western Australia 6845, Australia
}

(Received 12 June 2013; published 19 August 2013)

\begin{abstract}
The ionic compound cesium chloride adopts a cubic crystal structure bearing the same name. However, $a b$ initio electronic structure calculations based on density functional theory methods using generalized gradient approximation functionals do not predict that cesium chloride adopts this phase. In this paper we apply semiempirical methods (density functional theory plus a pairwise dispersion correction) to account for missing van der Waals interactions within cesium chloride. The $C_{6}$ and $R_{0}$ dispersion parameters for cesium are established within Grimme's DFT + D2 formalism. Inclusion of the dispersion corrections is found not only to improve the quality of structures in comparison to experiment for all cesium halides, but also leads to the correct prediction of the ground-state phase under ambient conditions.
\end{abstract}

DOI: 10.1103/PhysRevB.88.054112

PACS number(s): 31.15.E-, 61.66.Fn, 61.50.Lt, 71.20.-b

\section{INTRODUCTION}

It is widely assumed that the alkali-metal halides represent simple model systems that are well understood and require little further exploration by quantum mechanical methods. Most alkali-metal halides adopt the rocksalt $\mathrm{NaCl}$ (B1) structure, in which the coordination number is six. Only three compounds ( $\mathrm{CsCl}, \mathrm{CsBr}$, and $\mathrm{CsI}$ ), adopt the $\mathrm{CsCl}$ (B2) structure where the coordination number is eight. Under pressure the $\mathrm{B} 1$ alkali-metal halides transform to the $\mathrm{B} 2$ phase, ${ }^{1}$ while $\mathrm{CsCl}$ transforms from B2 to B1 above $742 \mathrm{~K}$ (Ref. 2). The relative stability between the B1 and B2 phases is textbook material and is conventionally explained using simple ionic models or radius ratio rules. ${ }^{3}$ It is therefore somewhat ironic that conventional density functional theory (DFT) calculations, based on the widely used generalized gradient approximation (GGA), do not predict that $\mathrm{CsCl}$ adopts the phase that bears its name, with instead the rocksalt B1 structure being favored. In this manuscript we explore this unexpected behavior, which is also shared by $\mathrm{CsBr}$ and $\mathrm{CsI}$.

The energetic balance between competing phases in the alkali-metal halides has been studied for the best part of a hundred years. As far back as the 1930s it was reported by Born and Mayer ${ }^{4,5}$ and London ${ }^{6}$ that an accurate description of the stability of the CsCl-type structure for $\mathrm{CsCl}, \mathrm{CsBr}$, and CsI required the treatment of both zero point energy effects and dispersion, with the latter contributing around $1 \%-5 \%$ of the lattice energy. Although a small fraction of the total binding energy, this difference may be sufficient to explain the transition from the $\mathrm{NaCl}$ - to $\mathrm{CsCl}$-type structure. Given that standard exchange-correlation functionals that depend only on the density and its first derivative at a single point in space are unable to capture the van der Waals (vdW) interactions that arise from dynamical correlation, this may explain this incorrect prediction.

There have been a number of theoretical studies of the competition between the B1-B2 structures for simple binary salts. ${ }^{7-14}$ Many of these studies have employed approximate methods, rather than a full quantum mechanical treatment. For example, the self-consistent atomic deformation method ${ }^{8}$ has been employed to compute the relative energy of these two structures for the alkali-metal halides. This approach was found to give a lower energy for the B1 structure in all cases. While this is correct for the majority of alkali-metal halides, it is naturally in error for $\mathrm{CsCl}, \mathrm{CsBr}$, and CsI. In the case of $\mathrm{CsCl}$, the B1 structure was favored over B2 by $0.10 \mathrm{eV}$ (Ref. 8). This is only a marginal quantitative improvement over a previous spherical ion result $(0.13 \mathrm{eV})$ obtained by Cortona. ${ }^{9}$ Further investigations examined whether treating the heavier Cs ions relativistically would improve the result, but this failed to alter the finding. ${ }^{10}$

Pyper has suggested ${ }^{11}$ that dispersion plays an important role in stabilizing the $\mathrm{B} 2$ phase in $\mathrm{CsCl}$, and his most recent calculations $^{12}$ using the relativistic integrals program ${ }^{15,16}$ predict that the $\mathrm{B} 2$ phase is $0.078 \mathrm{eV} /$ f.u. more stable than B1. The importance of dispersion has been questioned by Florez et al. ${ }^{13}$ who performed ab initio perturbed ion (AIPI) calculations ${ }^{17,18}$ on $\mathrm{CsCl}$ and found the correct phase ordering without taking explicit account of dispersion. However, these calculations incorrectly predicted that $\mathrm{RbF}$ and $\mathrm{CsF}$ also prefer the $\mathrm{B} 2$ structure, rather than the $\mathrm{B} 1$ phase seen experimentally. A later study on $\mathrm{CsCl}$ using the AIPI method and improved functionals by Aguado ${ }^{14}$ found an energy difference of approximately $0.14 \mathrm{eV} /$ f.u. in favor of B2, but the predicted lattice constant was $3 \%$ larger than the experimental value extrapolated to zero Kelvin.

Given the broad success and computational feasibility of DFT for many problems, the failure of many current exchange-correlation functionals to properly describe dispersion interactions has been a significant limitation, at least quantitatively. Consequently, there has been considerable recent interest in approaches that make some allowance for long-range dispersive interactions within the framework of a DFT calculation. One approach is to use an explicitly nonlocal exchange-correlation functional that depends on the density and its gradient simultaneously at two points in space. This allows the asymptotic two-body limit of a $1 / \mathrm{r}^{6}$ interaction to be recovered, though it should be noted that this is not appropriate for all systems. ${ }^{19-21}$ Alternatively, Grimme ${ }^{22}$ has advocated a 
semiempirical approach in which the dispersion contributions are additively included into a standard DFT calculation in the manner of an interatomic potential. Critical to this approach is that the dispersion contribution is damped at short range to avoid duplicating contributions that are captured by the exchange-correlation functional. A number of parametrizations have been proposed by Grimme for the combination of DFT calculations employing the generalized-gradient approximation (GGA) with supplementary dispersion terms. The original DFT + D (Ref. 23) and DFT + D2 (Ref. 22) employed empirical dispersion coefficients and interpolation formulas while the more recent DFT + D3 method (Refs. 24 and 25) uses an ab initio approach to calculate the dispersion coefficients. Of these various methods, the DFT + D2 approach is the most widely used. Recently the DFT + D3 method was privately implemented $^{26}$ but is not yet publicly available. Beyond these methods there are many alternative dispersion corrections, including those of Becke, Johnson, ${ }^{27,28}$ and co-workers who have derived schemes to determine the dispersion parameters for any system, including solids, from the properties of the exchange hole.

In this work we examine the influence of dispersion interactions on the relative energetics of the B1 and B2 structures using Grimme's DFT + D2 method. Currently the literature parametrization does not include elements from cesium onwards and so the appropriate dispersion parameters must first be derived. Following this we examine whether the lack of long-range dispersion interactions might indeed be the reason for the failure of current GGA calculations to correctly predict that cesium chloride should adopt the $\mathrm{CsCl}$ structure.

\section{METHODOLOGY}

All calculations have employed Kohn-Sham density functional theory (DFT) as implemented within the Vienna $a b$ initio simulation package (VASP). ${ }^{29,30}$ The effective potential due to the core electrons and nucleus combined was described using the projector augmented wave (PAW) method. ${ }^{31}$ The PAW potentials used here have nine valence electrons for $\mathrm{Cs}$ and seven valence electrons for $\mathrm{F}, \mathrm{Cl}, \mathrm{Br}$, and I. Convergence tests showed that kinetic energy cut-offs of $550 \mathrm{eV}$ for $\mathrm{CsF}$ and $500 \mathrm{eV}$ for $\mathrm{CsCl}, \mathrm{CsBr}$, and $\mathrm{CsI}$, were sufficient for the plane-wave expansion of the valence electron wave functions. The auxiliary grid that is used to expand the electron density and augmentation charges employed a kinetic energy cutoff that was four times that of the wave function. For the exchangecorrelation functional both the local density approximation (LDA) and generalized gradient approximation (GGA) of Perdew-Burke-Ernzerhof (PBE) $)^{32}$ were used. Sampling of the Brillouin zone was performed using a $k$-point mesh constructed according to the Monkhorst-Pack scheme. Here $k$-point meshes of $10 \times 10 \times 10$ and $6 \times 6 \times 6$ were used for the cesium chloride (B2) and rocksalt (B1) structures, respectively. During structural relaxation of each phase, all cell parameters and internal atomic positions were simultaneously relaxed. In all cases the total electronic energy was converged to better than $10^{-7} \mathrm{eV}$ within the self-consistent field, while the geometry optimization was considered to be converged when the energy change between minimization steps was less than $10^{-6} \mathrm{eV}$. Cohesive energies were determined by combining the bulk energy with spin-polarized atomic reference calculations for the constituent neutral species.

The long-range dispersion (van der Waals) contribution was described using the pragmatic DFT + D2 approach of Grimme, ${ }^{22}$ which involves adding a semiempirical dispersion term to the conventional Kohn-Sham ${ }^{33}$ DFT energy:

$$
E_{\mathrm{DFT}+\mathrm{D} 2}=E_{\mathrm{DFT}}+E_{\text {dispersion }} \text {. }
$$
form:

This dispersion contribution takes the following explicit

$$
E_{\text {dispersion }}=-s_{6} \sum_{i=1}^{N_{a t}-1} \sum_{j=i+1}^{N_{a t}} f\left(r_{i j}\right) \times \frac{C_{6}^{i j}}{r_{i j}^{6}},
$$

where $s_{6}$ is a global scaling factor that only depends on the density functional used (taking the value $s_{6}=0.75$ for PBE; Ref. 22), $N_{a t}$ is the number of atoms in the system, $C_{6}^{i j}$ is the dispersion coefficient for the atom pair $i j$, and $r_{i j}$ is the interatomic distance. The term $f\left(r_{i j}\right)$ is a damping function that avoids the $r \rightarrow 0$ singularity and also removes the contribution from the empirical dispersion term in the regions where the exchange-correlation contribution is large and thereby reduces duplication of any short-range van der Waals contributions. The function $f\left(r_{i j}\right)$ takes the form,

$$
f\left(r^{i j}\right)=\frac{1}{1+e^{-d\left(r^{i j} / R_{0}^{i j}-1\right)}},
$$

where $R_{0}^{i j}$ is the sum of the atomic vdW radii. In the DFT + D2 formalism the value of the damping parameter $d$ is 20.0 and the cutoff radius for the pairwise interactions is $30.0 \AA$. In order to facilitate general applicability without having to parametrize each specific interaction, the following combination rules are used to generate the pairwise dispersion coefficients $C_{6}^{i j}$ and vdW radii $R_{0}^{i j}$ :

$$
C_{6}^{i j}=\sqrt{C_{6}^{i} \cdot C_{6}^{j}}
$$

and

$$
R_{0}^{i j}=R_{0}^{i}+R_{0}^{j} .
$$

When the DFT + D2 method was proposed, the van der Waals parameters $C_{6}$ and $R_{0}$ were only determined for the elements of the first five rows of the periodic table. Accordingly, we first need to extend this parametrization to include appropriate values of $C_{6}$ and $R_{0}$ for $\mathrm{Cs}$, as will be described in the next section.

\section{RESULTS}

\section{A. Derivation of parameters}

There are several methods in the literature that have been used to compute the van der Waals parameters required for the empirical dispersion correction of density functional theory. For example, time-dependent density functional theory can be used to determine the properties of atoms within specific crystalline structures. ${ }^{34}$ In the present work we adhere to the structure-independent approach proposed by Grimme. In the original DFT $+\mathrm{D}$ scheme the atomic $C_{6}$ coefficients were taken from the work of $\mathrm{Wu}$ and $\mathrm{Yang}^{35}$ and averaged over the possible hybridization states of the atoms. The errors 
due to the use of atomic instead of hybridization-dependent $C_{6}$ coefficients were later estimated by Grimme ${ }^{22}$ to be on the order of $10 \%-20 \%$ of the binding energy. In order to obtain $C_{6}$ parameters for larger portions of the periodic table in a consistent manner, he proposed a simple computational scheme for atomic $C_{6}$ coefficients in the updated DFT $+\mathrm{D} 2$ version. In this new scheme, the $C_{6}$ parameter is derived from the London formula for dispersion and is based on the atomic ionization potentials $\left(I_{p}\right)$ and static dipole polarizabilities $(\alpha)$. The $C_{6}$ coefficient (in units of $\mathrm{J} \mathrm{nm}^{6} \mathrm{~mol}^{-1}$ ) for a single atom is then given as;

$$
C_{6}=0.05 \times N I_{p} \alpha,
$$

where $I_{p}$ and $\alpha$ are in atomic units (i.e., Hartrees and Bohr, respectively) and $N$ is the number of electrons for the noble gas atom from the same row. Accordingly, $N$ has the value 2, $10,18,36,54$, and 86 for atoms in rows 1 through 6 of the periodic table, respectively.

In Grimme's DFT + D2 scheme, the $C_{6}$ parameters were calculated based on DFT/PBE0 calculations of atomic ionization potentials $I_{p}$ and static dipole polarizabilities $\alpha$. To reproduce binding energies and bond lengths of the lighter elements and noble gas systems, the proportionality constant in Eq. (6) was adjusted. However, this approach provided a poor definition of $C_{6}$ for elements from Group I, Group II, and the transition metals due to significant differences between the free atom and the atom as found in typical bonding environments. Accordingly, Grimme chose to treat atoms in Groups I and II by averaging the $C_{6}$ coefficient of the preceding noble gas atom with the following Group-III element. Naturally, this approach cannot be employed for Cs as there is no subsequent Group-III value available.

In our approach to estimating $C_{6}$ for $\mathrm{Cs}$, we applied Eq. (6) to all Group-I elements using the first and second ionization potential $I_{p}$ and the corresponding atomic and ionic polarizabilities $(\alpha)$ to describe neutral and single ionized atoms, respectively. The resulting $C_{6}$ parameters are listed in Table I; also shown in the table are the $C_{6}$ parameters from the DFT + D2 scheme. ${ }^{22}$ A graphical comparison between the

TABLE I. Comparison between dispersion coefficients $C_{6}$ computed using Eq. (6) and available DFT +D2 values in Ref. 22. Calculated data for both neutral and singly ionized cations is shown. All $C_{6}$ values are expressed in units of $\mathrm{J} \mathrm{nm}^{6} \mathrm{~mol}^{-1}$. The quantity $N$ is defined in the text. Ionization potential data $\left(I_{p}\right)$ are from Ref. 36 . Polarizability data $(\alpha)$ are from Ref. 37.

\begin{tabular}{lcrrcc}
\hline \hline Species & $N$ & $\begin{array}{c}I_{p} \\
(\mathrm{eV})\end{array}$ & $\begin{array}{c}\alpha \\
(\text { a.u. })\end{array}$ & $\begin{array}{c}C_{6} \\
\text { (This work) }\end{array}$ & $\begin{array}{c}C_{6} \\
\text { (Grimme) }\end{array}$ \\
\hline $\mathrm{Li}$ & 10 & 5.39 & 163.98 & 16.25 & 1.61 \\
$\mathrm{Li}^{+}$ & 10 & 75.64 & 0.19 & 0.27 & \\
$\mathrm{Na}$ & 18 & 5.14 & 159.26 & 27.067 & 5.71 \\
$\mathrm{Na}^{+}$ & 18 & 45.28 & 1.00 & 1.57 & \\
$\mathrm{~K}$ & 36 & 4.34 & 292.87 & 84.09 & 10.80 \\
$\mathrm{~K}^{+}$ & 36 & 31.63 & 5.40 & 11.17 & \\
$\mathrm{Rb}^{+}$ & 54 & 4.18 & 319.19 & 132.28 & 24.67 \\
$\mathrm{Rb}^{+}$ & 54 & 27.29 & 9.08 & 24.57 & \\
$\mathrm{Cs}^{+}$ & 86 & 3.89 & 402.19 & 247.47 & \\
$\mathrm{Cs}^{+}$ & 86 & 23.11 & 15.81 & 57.74 & \\
\hline \hline
\end{tabular}

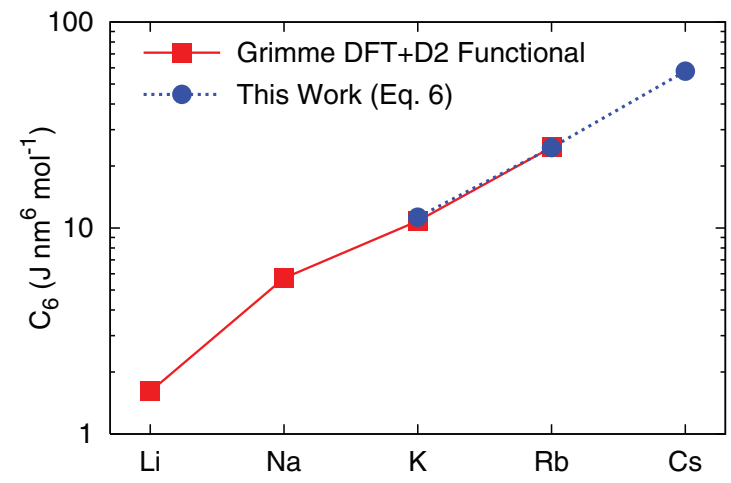

FIG. 1. (Color online) Comparison between selected Group-I dispersion coefficents calculated in this work and those in the Grimme $\mathrm{DFT}+\mathrm{D} 2$ functional. The values taken in the present work are those for ionized cations, as listed in Table I.

DFT + D2 values and our own computed values of $C_{6}$ for ionized cations is provided in Fig. 1. For the heavier cations $\left(\mathrm{K}^{+}\right.$and $\mathrm{Rb}^{+}$), our computed $C_{6}$ values for ionized cations are strikingly close to those reported by Grimme. This is an important observation as it provides a strategy for estimating $C_{6}$ for Cs. Also apparent from this table is a large difference in $C_{6}$ between the neutral and ionized cases, with the former being much higher than those reported by Grimme. This behavior is consistent with Grimme's observation that neutral atoms are an inappropriate reference point when calculating $C_{6}$ values from atoms in many bonding environments. For the lighter cations $\left(\mathrm{Li}^{+}\right.$and $\mathrm{Na}^{+}$) our calculated values deviate from those of Grimme, but this difference is peripheral to this work as the important behavior is the trend for heavy elements. Taking the second ionization potential of $\mathrm{Cs}\left(I_{p}=23.11 \mathrm{eV}\right)$ [Ref. 36] and the polarizability of the $\mathrm{Cs}^{+}$cation ( $\alpha=15.18$ a.u.) (Ref. 37 ), leads to a calculated value of $C_{6}=57.74 \mathrm{~J} \mathrm{~nm}^{6} \mathrm{~mol}^{-1}$ for Cs. This value is used throughout the remainder of this manuscript.

As a general comment we note that Fig. 1 shows the expected trend in which $C_{6}$ increases with atomic number due to the lower effective nuclear charge experienced by the valence electrons, making them more polarizable. As a result, dispersion plays an increasingly important role in the phase stability of compounds as one moves down Group I. An example of this importance can be seen in a recent DFT/GGA study of pressure-induced $(\mathrm{B} 1 \rightarrow \mathrm{B} 2)$ phase transitions in rubidium halides. ${ }^{38}$ The calculated transition pressures are significantly larger (typically by a factor of four) than experimental values, a discrepancy that may well be due to the absence of dispersion interactions in the DFT scheme. Indeed, in earlier interionic force calculations on the same system by Cohen and Gordon, ${ }^{39}$ they pointed out that if the dispersion contributions could be incorporated into their calculations, they would have been likely to have obtained better agreement with experiment.

In Grimme's DFT + D and DFT + D2 functionals the van der Waals radius employed in the damping function $\left(R_{0}\right)$ was determined for each element using the 0.01 a.u. electron density contour in ROHF/TZV calculations of atoms in their electronic ground state. This contour value was scaled by a factor of $1.22 \mathrm{in} \mathrm{DFT}+\mathrm{D}$ and $1.10 \mathrm{in} \mathrm{DFT}+\mathrm{D} 2$. To determine 

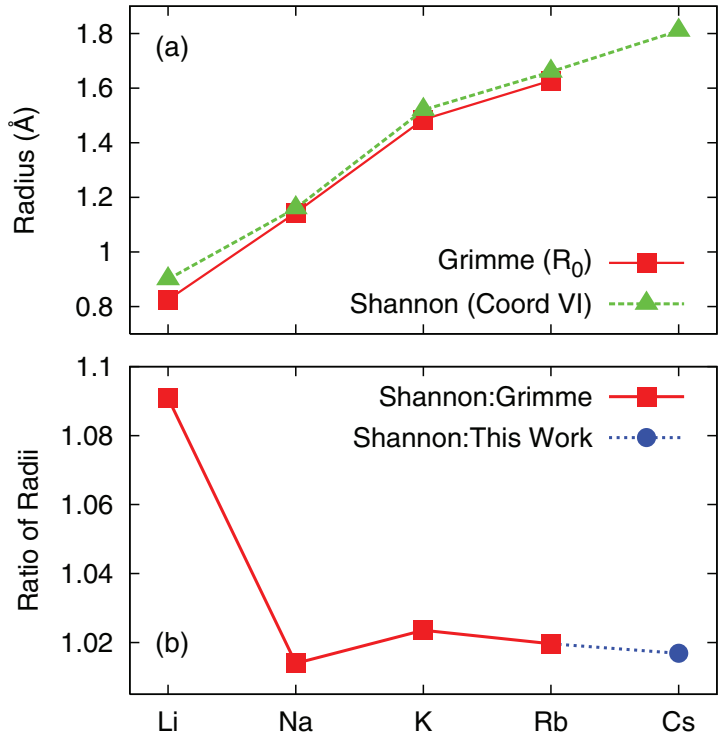

FIG. 2. (Color online) Graphical representation of (a) the data shown in Table II demonstrating the close relationship between the $R_{0}$ parameter in the DFT + D2 formalism (red squares) and the Shannon ionic radius for six-coordinate cations (green triangles), and (b) the ratio of the Shannon radii to Grimme $R_{0}$ values (red squares). The blue circle denotes the ratio corresponding to the estimated value of $R_{0}$ for Cs that is used in this work.

a value of $R_{0}$ for $\mathrm{Cs}$, we first compared the $R_{0}$ values for $\mathrm{Li}$, $\mathrm{Na}, \mathrm{K}$, and $\mathrm{Rb}$ from DFT $+\mathrm{D} 2$ against standard ionic radii. ${ }^{40}$ Since the ionic radius is a function of the coordination number, it is necessary to standardize on a consistent environment. Although the $\mathrm{CsCl}$ structure involves eightfold coordination, it is more reasonable to choose sixfold radii as a reference, given that octahedral environments predominate amongst the Group-I halides.

As shown in Fig. 2(a), there is a close correlation between the Shannon ionic radii and the values of $R_{0}$ used in the DFT + D2 method. Figure 2(b) and Table II present the ratio between $R_{0}$ and the six-coordinate Shannon ionic radius for the Group-I elements. It can be seen that the Shannon radii and Grimme $R_{0}$ values are largely close to each other with the former being slightly larger by a near constant ratio. In this regard, $\mathrm{Li}^{+}$is somewhat the exception with a marginally greater difference between the values. Excluding this element,

TABLE II. Comparison between the damping function parameter $\left(R_{0}\right)$ in the DFT $+\mathrm{D} 2$ approach $^{22}$ and Shannon ionic radii ${ }^{40}$ for six-coordinate cations. The DFT + D2 data are for $\mathrm{Li}-\mathrm{Rb}$, while the Shannon data are for Li-Cs. The value of $R_{0}$ for Cs is estimated as described in the text by scaling the Shannon radius by a factor of 1.019 .

\begin{tabular}{lccc}
\hline \hline Element & $R_{0}(\AA)$ & Shannon $(\mathrm{VI})(\AA)$ & Ratio \\
\hline $\mathrm{Li}$ & 0.825 & 0.90 & 1.091 \\
$\mathrm{Na}$ & 1.144 & 1.16 & 1.014 \\
$\mathrm{~K}$ & 1.485 & 1.52 & 1.024 \\
$\mathrm{Rb}$ & 1.628 & 1.66 & 1.020 \\
$\mathrm{Cs}$ & $1.776^{\mathrm{a}}$ & 1.81 & 1.019 \\
\hline \hline
\end{tabular}

${ }^{\mathrm{a}}$ This work

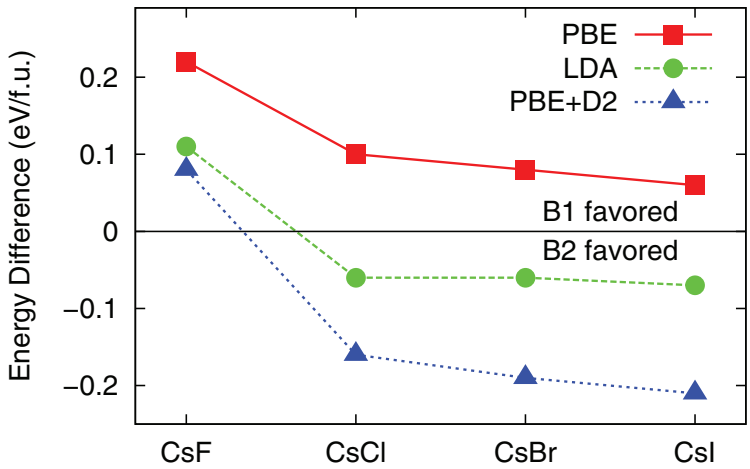

FIG. 3. (Color online) Energy differences (eV/f.u.) between the $\mathrm{B} 1$ and $\mathrm{B} 2$ phases of $\mathrm{CsX}(X=\mathrm{F}, \mathrm{Cl}, \mathrm{Br}, \mathrm{I})$ calculated using LDA (circles), $\mathrm{PBE}$ (squares), and $\mathrm{PBE}+\mathrm{D} 2$ (triangles) functionals.

the ratio of the Shannon ionic radii to Grimme $R_{0}$ is $1.019 \pm$ 0.005 for $\mathrm{Na}^{+}, \mathrm{K}^{+}$, and $\mathrm{Rb}^{+}$. Based on this average ratio for the heavier Group-I cations, we have estimated the $R_{0}$ value for Cs to be $1.776 \AA$. According to Grimme's philosophy, ${ }^{22}$ a Group-II element has the same $C_{6}$ and $R_{0}$ values as that of the Group-I element in the same row. Consequently, the parameters determined here for $\mathrm{Cs}$ can also be applied to barium.

\section{B. Application to cesium halides}

Using the fitted $C_{6}$ and $R_{0}$ parameters values for $\mathrm{Cs}^{+}$and the available parameters for the halogens, ${ }^{22}$ we performed both standard (LDA and PBE) and DFT + D2 dispersion-corrected PBE calculations for the cesium halides ( $\mathrm{CsF}, \mathrm{CsCl}, \mathrm{CsBr}$, and $\mathrm{CsI})$. In the following we will denote the latter calculations as PBE + D2 to emphasize the functional employed. Figure 3 shows the energy difference between the B1 and B2 phases of Cs halides as calculated at the LDA, PBE and PBE + D2 levels; the quantitative values are listed in Table III along with the cohesive energies. The energy difference $\Delta E$ is defined such that a positive value indicates the B1 structure is favored, while conversely a negative value indicates that B2 is favored. Although the LDA approach is generally less accurate than GGA, typically overbinding in comparison with experiment, here it successfully predicts the experimentally observed phase for all of the Cs halide compounds. The same is true for the $\mathrm{PBE}+\mathrm{D} 2$ calculations, which show a small systematic shift relative to the LDA energetics in favor of the B2 phase. At the PBE level, however, all four Cs halides are predicted to adopt the rocksalt $\mathrm{B} 1$ phase. In other words, calculations at the PBE/GGA level do not predict that $\mathrm{CsCl}$ adopts the $\mathrm{CsCl}$ structure. The same error is seen for $\mathrm{CsBr}$ and $\mathrm{CsI}$, which also adopt the B2 phase under ambient conditions. We note that our energy differences for $\mathrm{CsCl}$ using the $\mathrm{LDA}$ and $\mathrm{PBE}+\mathrm{D} 2$ functionals are similar to those calculated by Pyper ${ }^{12}$ and Aguado $^{14}$ who reported values of 0.078 and $0.14 \mathrm{eV} / \mathrm{f}$.u., respectively,

Cohesive energies calculated using the PBE + D2 functional (Table III) compare very well with experiment ${ }^{41}$ for the B2 phases where the difference is less than $0.16 \mathrm{eV} / \mathrm{f}$.u. This performance is superior to the other two functionals examined which have larger discrepancies; for LDA the maximum discrepancy for B2 phases is $0.30 \mathrm{eV} / \mathrm{f}$.u., while for PBE it is 
TABLE III. Cohesive energies (eV/f.u.) for the $\mathrm{B} 1$ and $\mathrm{B} 2$ phases of $\mathrm{CsX}(X=\mathrm{F}, \mathrm{Cl}, \mathrm{Br}$, I) calculated using LDA, PBE, and PBE + D2 functionals. The energy difference $\Delta E$ is defined in the text. The experimentally preferred phases are $\mathrm{B} 1$ for $\mathrm{CsF}$ and $\mathrm{B} 2$ for $\mathrm{CsCl}$, $\mathrm{CsBr}$, and CsI. This trend is reproduced by the LDA and PBE + D2 functionals, but not with PBE.

\begin{tabular}{|c|c|c|c|}
\hline Structures & $\mathrm{B} 1(\mathrm{NaCl})$ & $\mathrm{B} 2(\mathrm{CsCl})$ & $\Delta E$ \\
\hline \multicolumn{4}{|c|}{$\mathrm{CsF}$} \\
\hline LDA & -8.24 & -8.13 & 0.11 \\
\hline PBE & -7.19 & -6.97 & 0.22 \\
\hline $\mathrm{PBE}+\mathrm{D} 2$ & -7.94 & -7.86 & 0.08 \\
\hline Experiment $^{41}$ & -7.48 & & \\
\hline \multicolumn{4}{|c|}{$\mathrm{CsCl}$} \\
\hline LDA & -6.89 & -6.94 & -0.06 \\
\hline PBE & -6.13 & -6.04 & 0.10 \\
\hline $\mathrm{PBE}+\mathrm{D} 2$ & -6.68 & -6.84 & -0.16 \\
\hline Experiment $^{41}$ & & -6.74 & \\
\hline \multicolumn{4}{|c|}{$\mathrm{CsBr}$} \\
\hline LDA & -6.39 & -6.45 & -0.06 \\
\hline PBE & -5.68 & -5.60 & 0.08 \\
\hline $\mathrm{PBE}+\mathrm{D} 2$ & -6.29 & -6.48 & -0.19 \\
\hline Experiment ${ }^{41}$ & & -6.48 & \\
\hline \multicolumn{4}{|c|}{ CsI } \\
\hline LDA & -5.80 & -5.88 & -0.07 \\
\hline PBE & -5.13 & -5.07 & 0.06 \\
\hline $\mathrm{PBE}+\mathrm{D} 2$ & -5.81 & -6.02 & -0.21 \\
\hline Experiment ${ }^{41}$ & & -6.18 & \\
\hline
\end{tabular}

substantially greater at $1.11 \mathrm{eV} / \mathrm{f}$.u. In contrast, none of the functionals predict the cohesive energy particularly accurately for the B1 phase of CsF, with PBE being the closest.

The relative importance of dispersion can be quantified from Table III by comparing the cohesive energies of the $\mathrm{PBE}$ and $\mathrm{PBE}+\mathrm{D} 2$ calculations. This energy difference is surprisingly invariant, falling in the range of $0.55-0.75 \mathrm{eV} / \mathrm{f}$.u. for the B1 structures and $0.80-0.95 \mathrm{eV} / \mathrm{f}$.u. for the B2 structures; the average values are 0.65 and $0.88 \mathrm{eV} /$ f.u., respectively. There is no apparent trend down the group, suggesting that the increasing $C_{6}$ coefficient associated with a higher atomic number is counteracted by the larger lattice parameter. Accordingly, the dispersion contribution to the cohesive energy is roughly constant for a given structure type. We note that the average dispersion contribution in the B2 structures is larger than its B1 equivalent by a ratio of $8: 6$, which matches that of the coordination numbers of the two structures.

Although the dispersion force is comparatively weak, it is always attractive and hence its inclusion always decreases the cell volume. This behavior is evident in Fig. 4 which plots cell volumes for the B1 and B2 phases of the cesium halides at $0 \mathrm{~K}$. Experimental values for the cell volumes at $0 \mathrm{~K}$ were corrected from the lowest measured temperature ( $293 \mathrm{~K}$ for $\mathrm{CsF}, 5 \mathrm{~K}$ for $\mathrm{CsCl}$, and $20 \mathrm{~K}$ for $\mathrm{CsBr}$ and $\mathrm{CsI}$ ) using experimental thermal expansion data. ${ }^{42,43}$ In the case of the $\mathrm{B} 1-\mathrm{CsCl}$ phase the experimental value at $450 \mathrm{~K}$ was corrected to $0 \mathrm{~K}$ by assuming that this phase has the same thermal expansion coefficient as the B2 phase. Whereas the PBE functional consistently overestimates the volume (by an average of 9\%), the LDA
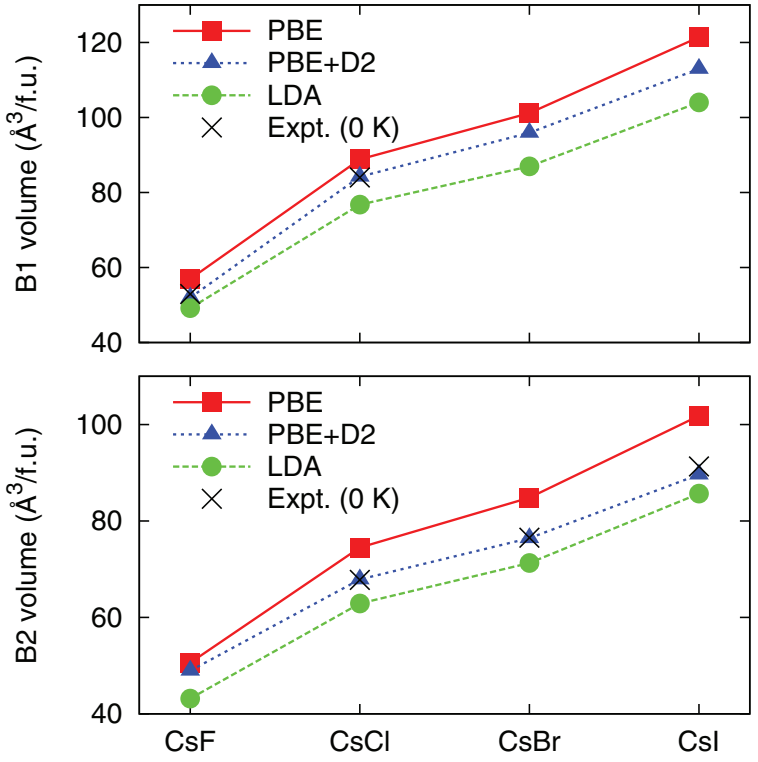

FIG. 4. (Color online) Cell volumes ( $\AA^{3} /$ f.u.) for the B1 and B2 phases of $\mathrm{CsX}(X=\mathrm{F}, \mathrm{Cl}, \mathrm{Br}, \mathrm{I})$ calculated using LDA (circles), PBE (squares), and PBE + D2 (triangles) functionals. Experimental values (crosses) for the cell volumes were corrected from the lowest measured temperature to $0 \mathrm{~K}$ using the available thermal expansion data (see text for details).

functional conversely underestimates the same quantity (by an average of $7 \%$ ). In every case the PBE + D2 functional provides an excellent prediction of the cell volume with an average difference of only $0.9 \%$. Furthermore, the PBE + D2 results are far closer to the extrapolated experimental values at $0 \mathrm{~K}$ than those obtained in calculated by Aguado ${ }^{14}$ and Pyper ${ }^{12}$ for which the cell volume at zero Kelvin is overestimated by 8.7 and $5.3 \%$, respectively.

\section{Sensitivity analysis}

Given that the parameters $C_{6}$ and $R_{0}$ are empirically determined, it is instructive to perform a sensitivity analysis of their effects. For this we have chosen to focus on the compound $\mathrm{CsCl}$, using the lattice parameter of the $\mathrm{B} 2$ phase and the energy difference between the B1 and B2 phases to highlight the effect of varying $C_{6}$ (Fig. 5) and $R_{0}$ (Fig. 6). The vertical dashed line in each figure indicates the parameter value used elsewhere in this work. Also included in Figs. 5(a) and 6(a) using solid horizontal lines are the experimental lattice parameter at both $300 \mathrm{~K}$ and $0 \mathrm{~K}$, which is corrected using the available thermal expansion data, and the value computed by Pyper. ${ }^{12}$ The B1-B2 energy difference calculated by Pyper $^{12}$ and Aguado ${ }^{14}$ is also shown, with the latter being closer to our PBE $+\mathrm{D} 2$ result of $0.16 \mathrm{eV} /$ f.u. Both the lattice parameter and the B1-B2 energy difference demonstrate great sensitivity to the choice of $C_{6}$ and $R_{0}$ parameters, as would be expected. When either $C_{6}$ increases or $R_{0}$ decreases, the dispersion forces become stronger, which increases the binding in $\mathrm{CsCl}$ and makes the $\mathrm{B} 2$ phase more stable than the B1 structure. Our $C_{6}$ and $R_{0}$ parameters for $\mathrm{Cs}$ in the $\mathrm{DFT}+\mathrm{D} 2$ calculations are obtained independently and rely only on the physical properties of the Cs cation. By employing these parameter values, the calculated cell lattice parameter of 


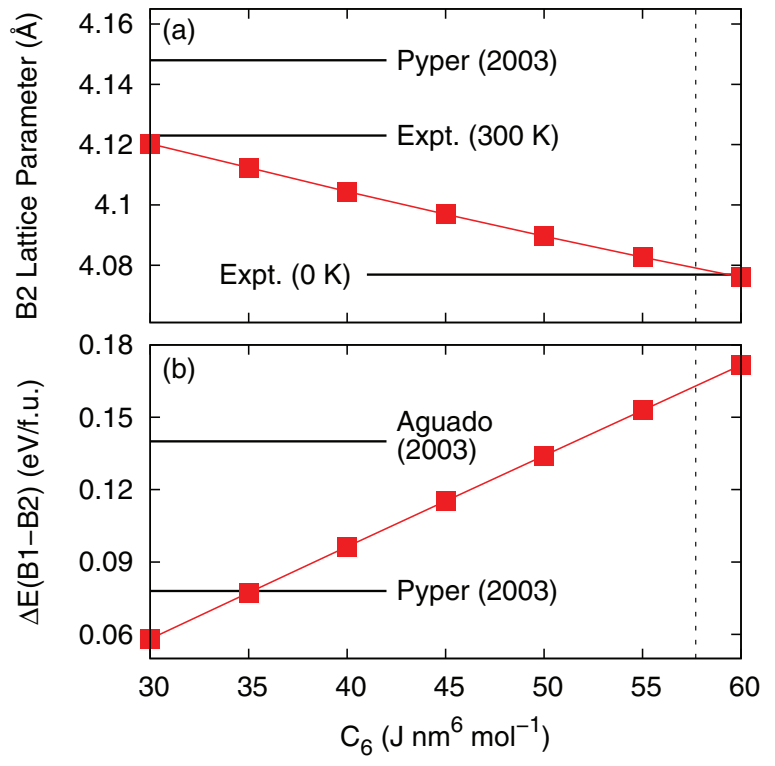

FIG. 5. (Color online) $C_{6}\left(\mathrm{~J} \mathrm{~nm}^{6} \mathrm{~mol}^{-1}\right)$ sensitivity analysis for (a) $\mathrm{CsCl}$ lattice parameter $(\AA)$ and (b) its energy difference between $\mathrm{B} 1$ and $\mathrm{B} 2$ phases $\Delta E(\mathrm{eV} /$ f.u.). Also shown are experimental lattice parameters at $0 \mathrm{~K}$ and $300 \mathrm{~K}$, and the values of (a) the lattice parameter and (b) the energy difference computed by Pyper. ${ }^{12}$ Panel (b) also shows the energy difference computed by Aguado ${ }^{14}$ but for clarity the predicted B2 lattice parameter of $4.192 \AA$ is not shown in panel (a). The experimental value at $0 \mathrm{~K}$ is obtained as described in the text. $R_{0}$ is fixed as $1.776 \AA$. The dashed vertical line represents the value $\left(57.74 \mathrm{~J} \mathrm{~nm}^{6} \mathrm{~mol}^{-1}\right)$ we used in this work.

$\mathrm{B} 2-\mathrm{CsCl}$ happens to be very close to that of the experimental value extrapolated to $0 \mathrm{~K}$. While this represents a useful further validation of our choice of parameters, it should be noted that

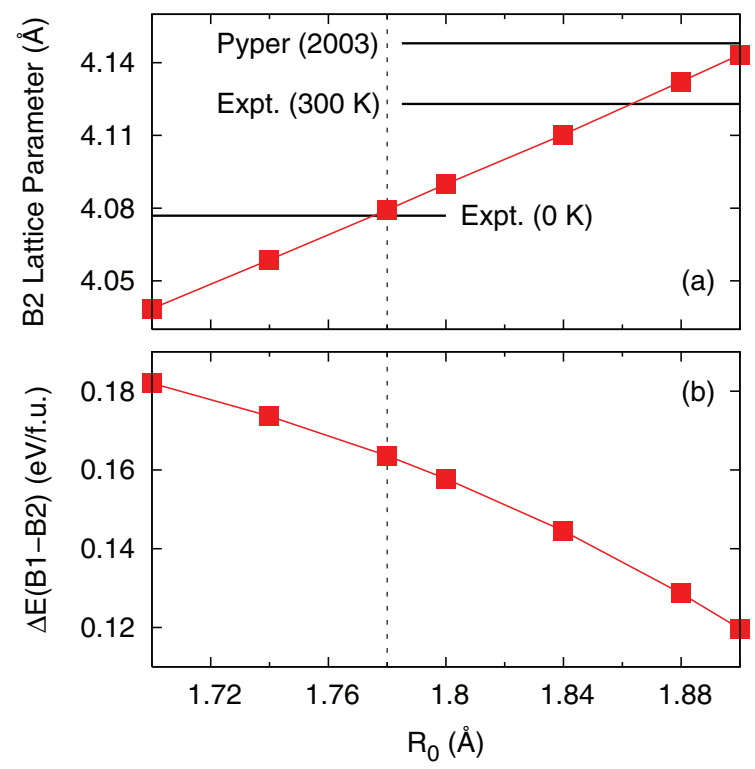

FIG. 6. (Color online) $R_{0}(\AA)$ sensitivity analysis for (a) $\mathrm{CsCl}$ lattice parameter $(\AA)$ and (b) its energy difference between B1 and B2 phases $\Delta E$ (eV/f.u.). Also shown are experimental lattice parameters at $0 \mathrm{~K}$ and $300 \mathrm{~K}$, and the values computed by Pyper. ${ }^{12} C_{6}$ is fixed at $57.74 \mathrm{~J} \mathrm{~nm}^{6} \mathrm{~mol}^{-1}$. The dashed vertical line indicates the value $(1.776 \AA)$ used in this work. the DFT + D2 should formally be corrected for zero point energy effects in order to equate it to the experimental value at absolute zero. However, for the relatively heavy atoms present in $\mathrm{CsCl}$ it is unlikely that the zero point vibration will lead to a substantial expansion of the unit cell.

An alternative strategy to obtain values for $R_{0}$ and $C_{6}$ would have been to fit the parameters to the available experimental properties. However, given that the present systematic approach has managed to deliver both physically reasonable structures and phase stabilities, this would have been less satisfying and may have led to a less transferable parametrization.

\section{DISCUSSION AND CONCLUSIONS}

In this work, we have examined the failure of density functional theory based on a widely used generalized gradient approximation, namely $\mathrm{PBE}$, to correctly predict that $\mathrm{CsCl}$ should adopt the cesium chloride structure at standard conditions. Although not exhaustively examined, we strongly suspect that this failure will also occur with other GGA exchange-correlation functionals in the literature. Given that the local density approximation yields the correct result, the overestimation of volume is clearly correlated with the error in the relative phase energies. As nearly all GGA functionals similarly underestimate the binding of solids, it appears reasonable that the failure will be widespread. It might be expected that the family of GGA functionals that are specifically parametrized for solids or from the Airy gas, such as Wu-Cohen ${ }^{44}$ and AM05, ${ }^{45}$ respectively, could give improved results. Hence we have tested the performance of a solid-state parameterized GGA, namely PBEsol, ${ }^{46}$ for the B1-B2 energy difference in the case of $\mathrm{CsCl}$. While the relative energy is reduced to $0.03 \mathrm{eV} / \mathrm{f}$.u. with this functional, as compared to $0.10 \mathrm{eV} /$ f.u. from $\mathrm{PBE}$, the result remains qualitatively incorrect.

As a result of the erroneous predictions arising from the use of GGA functionals alone, we have examined the influence of including semiempirical long-range dispersion via the DFT + D2 methodology of Grimme and co-workers. Although the dispersion energy corrections are small as a fraction of the overall cohesive energy, we find that it plays a vital role in determining the phase stability of $\mathrm{CsCl}, \mathrm{CsBr}$, and $\mathrm{CsI}$. The importance of the dispersion correction can also be seen in the results of calculations using a wide range of functionals, including PBEsol, the screened hybrid, HSE06 (Ref. 47), and also the hybrid functional, PBE0 (Ref. 48). Table IV presents the energy difference $\Delta E$ between $\mathrm{B} 1$ - and $\mathrm{B} 2$-type $\mathrm{CsCl}$ as

TABLE IV. The energy difference $\Delta E(\mathrm{eV} /$ f.u. $)$ between B1and $\mathrm{B} 2$-type $\mathrm{CsCl}$ calculated by using the exchange-correlation functionals PBEsol, HSE06, and PBE0. $\Delta E$ is defined as B2-B1, such that a positive value indicates the $\mathrm{B} 1$ structure is favored, while conversely a negative value indicates that $\mathrm{B} 2$ is favored.

\begin{tabular}{lcccr}
\hline \hline \multicolumn{2}{l}{ Functional } & PBEsol & HSE06 & PBE0 \\
\hline \multirow{2}{*}{$\Delta E$} & Without D2 & 0.028 & 0.082 & 0.077 \\
& With D2 & -0.194 & -0.225 & -0.231 \\
\hline \hline
\end{tabular}


determined using PBEsol, HSE06, and PBE0. All of these functionals exhibit the same qualitative result as for PBE, i.e., the incorrect order of stability for $\mathrm{CsCl}$. Addition of dispersion corrections to any of these functionals leads to the correct order of phase stability, thereby showing that dispersion is indeed the single most important factor.

In the present work the $C_{6}$ and $R_{0}$ parameters for cesium in the dispersion correction have been empirically determined based on a combination of theoretical arguments and extrapolation of data for the alkali-metal cations. Importantly, no specific reference is made to the target cesium halide structures to which the parameters have been applied. Despite this, and the demonstrated sensitivity of the results to the choice of dispersion parameters, the values determined here (i.e., $57.74 \mathrm{~J} \mathrm{~nm}^{6} \mathrm{~mol}^{-1}$ for $C_{6}$ and $1.776 \AA$ for $R_{0}$ ) give not only the correct relative phase stabilities, but also superior lattice parameters relative to the other functionals examined. With one exception, the cohesive energies are also in improved agreement with the experimental values. Finally, we also note that the magnitude of the dispersion energy contribution for the cesium halides does not exhibit a systematic variation with size or atomic number of the ions, although the underlying $C_{6}$ and $R_{0}$ parameters obviously do. It appears that the increased lattice parameter for larger ions offsets the corresponding increase in polarizability. Instead, the crucial factor that corrects the relative stability of the $\mathrm{B} 1$ and $\mathrm{B} 2$ phases is that the total dispersion energy is found to be proportional to the first coordination number of the structure, which is sufficient for $\mathrm{CsCl}$ to favor the cesium chloride structure, just as we would expect.

\section{ACKNOWLEDGMENTS}

We gratefully acknowledge financial support from the US Department of Energy through the LANL/LDRD program. J.D.G. and N.A.M. both thank the Australian Research Council for fellowships and iVEC and National Computational Infrastructure for provision of computing resources. *f.zhang@ curtin.edu.au

${ }^{1}$ C. W. Pistorius, Prog. Solid State Chem. 11, 1 (1976).

${ }^{2}$ J. Shanker and M. Kumar, Phys. Status Solidi (b) 158, 11 (1990).

${ }^{3}$ W. B. Jensen, Journal of Chemical Education 87, 587 (2010).

${ }^{4}$ M. Born and J. E. Mayer, Zeitschrift für Physik A Hadrons and Nuclei 75, 1 (1932).

${ }^{5}$ J. E. Mayer, J. Chem. Phys. 1, 270 (1933).

${ }^{6}$ F. London, Trans. Faraday Soc. 33, 8b (1937).

${ }^{7}$ J. Narain, N. K. Dwivedi, G. G. Agrawal, and J. Shanker, Phys. Status Solidi (b) 132, 389 (1985).

${ }^{8}$ W. N. Mei, L. L. Boyer, M. J. Mehl, M. M. Ossowski, and H. T. Stokes, Phys. Rev. B 61, 11425 (2000).

${ }^{9}$ P. Cortona, Phys. Rev. B 46, 2008 (1992).

${ }^{10}$ J. M. Recio, A. M. Pendás, E. Francisco, M. Flórez, and V. Luaña, Phys. Rev. B 48, 5891 (1993).

${ }^{11}$ N. C. Pyper, Chem. Phys. Lett. 220, 70 (1994).

${ }^{12}$ N. C. Pyper, J. Chem. Phys. 118, 2308 (2003).

${ }^{13}$ M. Flórez, J. M. Recio, E. Francisco, M. A. Blanco, and A. M. Pendás, Phys. Rev. B 66, 144112 (2002).

${ }^{14}$ A. Aguado, J. Chem. Phys. 119, 8765 (2003).

${ }^{15}$ C. Wood and N. Pyper, Chem. Phys. Lett. 81, 395 (1981).

${ }^{16}$ C. Wood and N. Pyper, Philos. Trans. R. Soc. A: Physical, Mathematical and Engineering Sciences 320, 71 (1986).

${ }^{17}$ V. Luaña and L. Pueyo, Phys. Rev. B 41, 3800 (1990).

${ }^{18}$ M. A. Blanco, A. M. Pendás, and V. Luaña, Comput. Phys. Commun. 103, 287 (1997).

${ }^{19}$ G. Román-Pérez and J. M. Soler, Phys. Rev. Lett. 103, 096102 (2009).

${ }^{20}$ M. Dion, H. Rydberg, E. Schröder, D. C. Langreth, and B. I. Lundqvist, Phys. Rev. Lett. 92, 246401 (2004).

${ }^{21}$ J. F. Dobson, A. White, and A. Rubio, Phys. Rev. Lett. 96, 073201 (2006).

${ }^{22}$ S. Grimme, J. Comp. Chem. 27, 1787 (2006).

${ }^{23}$ S. Grimme, J. Comput. Chem. 25, 1463 (2004).

${ }^{24}$ S. Grimme, J. Antony, S. Ehrlich, and H. Krieg, J. Chem. Phys. 132, 154104 (2010).
${ }^{25}$ S. Grimme, S. Ehrlich, and L. Goerigk, J. Comput. Chem. 32, 1456 (2011).

${ }^{26}$ W. Reckien, F. Janetzko, M. F. Peintinger, and T. Bredow, J. Comput. Chem. 33, 2023 (2012).

${ }^{27}$ A. D. Becke and E. R. Johnson, J. Chem. Phys. 122, 154104 (2005).

${ }^{28}$ A. D. Becke and E. R. Johnson, J. Chem. Phys. 127, 154108 (2007).

${ }^{29}$ G. Kresse and J. Furthmüller, Phys. Rev. B 54, 11169 (1996).

${ }^{30}$ G. Kresse and J. Furthmüller, Comput. Mater. Sci. 6, 15 (1996).

${ }^{31}$ P. E. Blöchl, Phys. Rev. B 50, 17953 (1994).

${ }^{32}$ J. P. Perdew, K. Burke, and M. Ernzerhof, Phys. Rev. Lett. 77, 3865 (1996).

${ }^{33}$ W. Kohn and L. J. Sham, Phys. Rev. 140, A1133 (1965).

${ }^{34}$ G.-X. Zhang, A. Tkatchenko, J. Paier, H. Appel, and M. Scheffler, Phys. Rev. Lett. 107, 245501 (2011).

${ }^{35}$ Q. Wu and W. Yang, J. Chem. Phys. 116, 515 (2002).

${ }^{36}$ J. E. Huheey, Inorganic Chemistry: Principles of Structure and Reactivity, 3rd ed. (Harper and Row, New York, 1983).

${ }^{37}$ S. Hati and D. Datta, J. Phys. Chem. 99, 10742 (1995).

${ }^{38}$ K. Haddadi, A. Bouhemadou, L. Louail, and D. Maouche, Phase Transitions 82, 266 (2009).

${ }^{39}$ A. J. Cohen and R. G. Gordon, Phys. Rev. B 12, 3228 (1975).

${ }^{40}$ R. D. Shannon, Acta Crystallographica Section A 32, 751 (1976).

${ }^{41}$ M. Tosi, Journal of Physics and Chemistry of Solids 24, 965 (1963).

${ }^{42}$ G. K. White and J. G. Collins, Proc. R. Soc. London A Mathematical and Physical Sciences 333, 237 (1973).

${ }^{43}$ K. Wang and R. Reeber, J. Appl. Crystallogr. 28, 306 (1995).

${ }^{44}$ Z. Wu and R. E. Cohen, Phys. Rev. B 73, 235116 (2006).

${ }^{45}$ A. E. Mattsson and R. Armiento, Phys. Rev. B 79, 155101 (2009).

${ }^{46}$ J. P. Perdew, A. Ruzsinszky, G. I. Csonka, O. A. Vydrov, G. E. Scuseria, L. A. Constantin, X. Zhou, and K. Burke, Phys. Rev. Lett. 100, 136406 (2008).

${ }^{47}$ A. V. Krukau, O. A. Vydrov, A. F. Izmaylov, and G. E. Scuseria, J. Chem. Phys. 125, 224106 (2006).

${ }^{48}$ C. Adamo and V. Barone, J. Chem. Phys. 110, 6158 (1999). 